Communications in Physics, Vol. 30, No. 1 (2020), pp. 79-84

DOI:10.15625/0868-3166/30/1/14439

\title{
TWO-COLOUR THREE-PULSE SPECTRALLY RESOLVED NONLINEAR SPECTROSCOPY FOR STUDIES OF DYNAMICS IN SEMICONDUCTOR Si QUANTUM DOTS
}

\author{
VUONG VAN CUONG ${ }^{1}$, DINH BA KHUONG ${ }^{2,3, \dagger}$, DAO VAN LAP $^{3}$ \\ ${ }^{1}$ Hanoi National University of Education, Hanoi, Vietnam \\ ${ }^{2}$ University of Science and Technology, University of Danang, Vietnam \\ ${ }^{3}$ Centre for Quantum and Optical Science, \\ Swinburne University of Technology, Melbourne, Australia \\ ${ }^{\dagger} E$-mail: kdinh@swin.edu.au
}

Received 23 September 2019

Accepted for publication 17 January 2020

Published 28 February 2020

\begin{abstract}
We demonstrate that a two-colour three-pulse nonlinear spectroscopy can be used to study the dynamics of excited carriers in Si quantum dot structures embedded in SiN. Decays of the transverse optical phonon population and the transverse acoustic phonon population are measured and discussed. A theoretical simulation is also performed to support interpretation of our experimental observations.
\end{abstract}

Keywords: Ultrafast spectroscopy; Laser spectroscopy; Optical properties of quantum dots.

Classification numbers: 78.47.J-; 42.62.Fi; 78.67.Hc.

\section{INTRODUCTION}

Ultrafast spectroscopy has become a fundamental tool that allows us to manipulate and probe the temporal evolution of a quantum system. Since the initial pioneering work of Ahmed Zewail [1], there have been various ultrafast spectroscopy techniques, such as the pump-probe technique [2], time resolved photoluminescence [3], four-wave mixing, and photon echoes [4,5] which have been proposed to explore important insights from molecular dynamics, e.g., population lifetimes, decoherence times, vibrational relaxation times, and coherence coupling.

The field of ultrafast spectroscopy of semiconductors continues to be an active field of research that allows us to further investigate fundamental phenomena in semiconductor physics and in many optoelectronic as well as electronic devices. Particularly, multi-dimensional coherent (C)2020 Vietnam Academy of Science and Technology 
nonlinear spectroscopy techniques [6] can be employed to offer a comprehensive understanding of ultrafast carrier dynamics in graphene [7], the dynamical evolution of excitons in semiconductor quantum dots and semiconductor quantum wells [8]. Recently, by using this technique, Marten Richter et al. have extracted the many-particle diffusion Green's functions for excitons in a semiconductor quantum well from multi-dimensional coherent spectra [9]. This technique has been also applied to investigate the femtosecond dynamics upon the electronic excitation of cold, weakly-bound molecules, and the induced dynamics from the interaction with the helium environment $[10,11]$.

The multidimensional spectroscopy technique relies on a four-wave mixing signal which is resulted from interaction of a sequence of ultrafast optical pulses with a nonlinear medium. We recall that interaction of the three pulses creates a third-order nonlinear response which acts as a driving force for the generation of the four-wave mixing field. Based on the four-wave mixing process, the optical response can be clearly unravelled onto multiple frequency axes by correlating the phase evolution of the four-wave mixing signal across two or three time periods and taking a multi-dimensional Fourier transform. When quantum dots, which are fragments of matter smaller than the electronic Bohr radius in all three coordinates, with a size normally ranging from a few hundreds to many hundreds of atoms are built from direct-gap semiconductors, e.g., InAs or GaAs, the low-temperature photoluminescence is analysed by sharp spikes to confirm the atomic-like nature of the emitting states. On the other hand, in the case of indirect-gap semiconductor quantum dots (QDs), e.g., silicon, the emission and absorption spectra become more complicated. For indirect-gap bulk materials, because of momentum conservation, purely electronic optical transitions at the band edge are not allowed to take place and therefore only phonon-assisted transitions can occur. The multi-phonon broadening which is associated with low acoustic-phonon modes is considerable. Consequently, the total broadening is large leading to the fact that sharp spikes are not observed in the emission and absorption spectra of small silicon QDs. Moreover, the relative contributions and influence of zero-phonon and phonon-assisted transitions on the optical properties of Si QDs have not been extensively studied in previous studies. It is worth recalling that because Si QDs are currently finding wide application, e.g., solar cells [12,13], study of Si QDs still attracts a lot of attention in the scientific community.

In this paper we demonstrate the possibility of using a spectrally resolved two-colour threepulse four-wave mixing process to study ultrafast dynamics in semiconductor Si quantum dots. In particularly, the time evolution of the nonlinear signals which are generated by the interaction of three driving laser pulses with wavelengths $\lambda_{1}=\lambda_{2}$, and the wavelength $\lambda_{3}$ ( $\lambda_{3}$ can be different to or the same as $\lambda_{1,2}$ ) is analyzed. A very short dephasing time and ultrafast decay times of "hot" excited carriers and the contribution of optical phonon modes in the carrier dynamics are then discussed. Using a simple theoretical model for a coupled two level system, we qualitatively interpret our experimental observations.

\section{EXPERIMENT}

A $800 \mathrm{~nm}, 1 \mathrm{~mJ}, 80 \mathrm{fs}, 1 \mathrm{kHz}$ repetition-rate laser beam which is generated by a modelocked Ti:sapphire laser and a regenerative amplifier is used for our experiments. The fundamental laser beam is then split into two beams for pumping two independent optical parametric amplifiers (OPAs). By using two optical crystals which are BBO type I and BBO type II, a broad range of wavelengths $(250-2000 \mathrm{~nm})$ with a pulse duration of about $100 \mathrm{fs}$ and the energy of 1-30 $\mu \mathrm{J}$ can 
be produced when different frequency conversion processes of the signal or idler pulse and the fundamental beam are applied (second harmonic generation, fourth-harmonic generation or sum frequency generation) $[14,15]$. The output beam from the first OPA is split into two beams, $\lambda_{1}$ and $\lambda_{2}$. The second OPA generates the third pulse $\lambda_{3}$. The first pulse $\lambda_{1}$ then passes through a translation stage to provide a delay relative to the second pulse $\lambda_{2}$ by a designated time $t_{12}$. The third pulse $\lambda_{3}$ passes through a second translation stage to provide a delay relative to the second pulse, designated by $t_{23}$. The three pulses with parallel polarizations are aligned parallel to each other and then are focused by a $15 \mathrm{~cm}$ focal length lens into the sample. On the surface of the lens, the three pulses form a triangular configuration with approximately $1 \mathrm{~cm}$ size. To ensure that there are transitions between the energy states of the QDs, the wavelength range of three beams is chosen from $500 \mathrm{~nm}$ to $650 \mathrm{~nm}$. The energy of the excitation pulses is kept as low as possible to minimise intensity dependent effects. The signal is generated from the sample in the phasematching direction $k_{4}=-k_{1}+k_{2}+k_{3}$ and is captured simultaneously by a spectrometer equipped with double charge-coupled device (CCD) arrays having a spectral resolution of $1 \mathrm{~nm}$.

In this study, we use a sample consisting of five bilayers of Si quantum dot superlattices embedded in a sapphire substrate, with an average dot size distribution of $4.3 \mathrm{~nm}$ and the dot density of $10^{12}-10^{13}$ dots $/ \mathrm{cm}^{3}$. The Si QDs were simply fabricated by using a RF $(13.56 \mathrm{MHz})$ driven plasma enhanced chemical vapour deposition (PECVD). In this fabrication process, silane, ammonia and nitrogen gas at a purity of $99.9999 \%$ were employed to act as the reactant gas sources and their flow rates were controlled to modulate the growth rate of the silicon nitride and siliconrich nitride (SRN) film. It is useful to mention that the flow rates of silane, ammonia and nitrogen were in the range 1 to $5 \mathrm{sccm}, 0$ to $10 \mathrm{sccm}$ and 300 to $500 \mathrm{sccm}$, respectively. P-type CZ (100) crystalline silicon wafers were used as sample substrates. A deposition pressure of 300 mTorr, a RF plasma power of $5 \mathrm{~W}$ and a growth temperature of $400^{\circ} \mathrm{C}$ were fixed during the process. The stoichiometry of the films was determined by the ratio of gas mixture. Silicon precipitations associated with the diffusion of $\mathrm{Si}$ atoms leaving the stoichiometric nitride (Si3N4) during high temperature thermal annealing were generated by the silicon-rich nitride (SRN) which consists of an excess of silicon in sub-stoichiometric nitride. We recall that this mechanism is similar to that for Si quantum dot formation in silicon-rich oxide (SRO) [16]. The conventional furnace annealing under a nitrogen atmosphere were conducted to precipitate $\mathrm{Si}$ and nanocrystalline $\mathrm{Si}$ growth by the diffusion of silicon in the oxide.

\section{RESULTS AND DISCUSSION}

In order to study the carrier population dynamics which can reveal the relaxation time of excited carriers, we investigate the time evolution of signal spectra which are obtained by varying the population times $t_{23}$ at fixed coherence delay $t_{12}$ of $0 \mathrm{fs}$. Fig. 1a shows the measured spectrum for semiconductor Si QDs when a very short population time is varied up to $\sim 600 \mathrm{fs}$ and the pump and the probe wavelengths are the same, i.e., $\lambda_{1,2}=\lambda_{3}=600 \mathrm{~nm}$. By using this wavelength, it is expected that the laser pulse generates carriers predominantly around the band edge. For a qualitative interpretation of the experimental observations, we perform the theoretical simulation which is shown in Fig. 1b. In this simulation, a simple two electronic state system with ground state $|g\rangle$ and excited state $|e\rangle$ is considered. The excited state consists of two levels $|e\rangle$ and $|e l\rangle$ corresponding to two transition modes with wavelengths $\lambda_{e g}$ and $\lambda_{e^{\prime} g}\left(\lambda_{e g}<\lambda_{e^{\prime} g}\right)$. The energy 
difference of the two modes is small $(<10 \mathrm{meV})$ and therefore the excited laser can easily span the two modes. In addition, because the two excited levels are coupled to a same ground electronic state $|g\rangle$, coherence transfer between these states can occur.
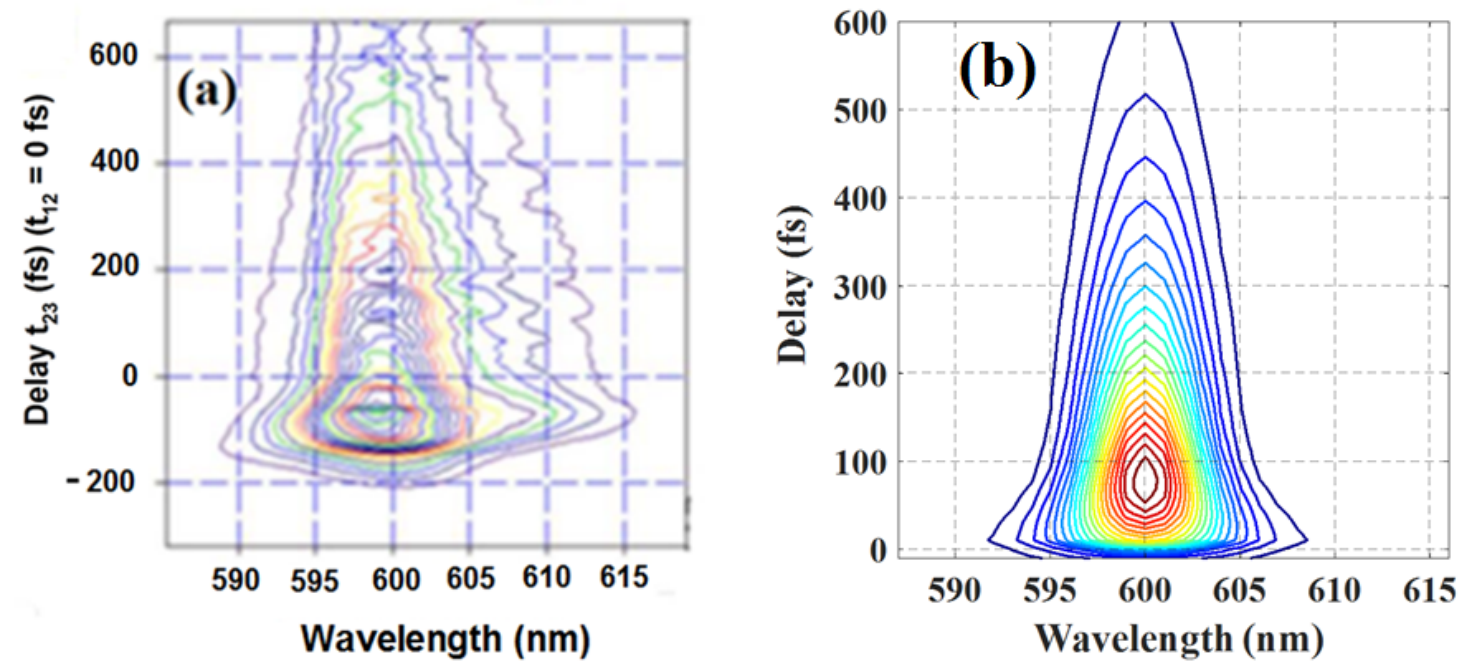

Fig. 1. Measured spectrum (a) and simulated spectrum (b) of three-pulse nonlinear signals $\left(\lambda_{1,2}=\lambda_{3}=600 \mathrm{~nm}\right)$ for Si QDs versus delay time $t_{23}$ up to $600 \mathrm{fs}$ when $t_{12}=0$.

In Figure 1a, it is clear that there is a broadening on both sides of the spectrum at short population times. This experimental observation is in good agreement with the simulation shown in Figure 1b. This indicates a significant contribution of the nonlinear signal to the signal spectrum.

For further analysis of our experimental observations, the spectrum for a long scan of population time, $t_{23}$, is measured. The time evolution of the signal spectrum detected in this experiment is plotted in Figure 2a. The corresponding simulated spectrum is shown in Figure $2 b$.

By fitting experimental data using two exponentials with a very long decay ( $>50 \mathrm{fs}$ ) as an offset, we find that there is a shorter decay which occurs with a lifetime of approximately 400 fs and the lifetime for the second decay component is around $7.5 \mathrm{ps.} \mathrm{These} \mathrm{measurements} \mathrm{are}$ consistent with our previous works [15].

It is worth noting that in crystalline $\mathrm{Si}$, three different phonon modes consisting of the transverse optical (TO), the longitudinal optical (LO) and the transverse acoustic (TA) modes contribute to the optical properties [17]. Particularly, the TO modes dominate the phonon-assisted optical transitions in weakly confined nanocrystalline $\mathrm{Si}$ with small contributions from LO and TA phonons. Previous works have shown that the ratio of the transition probabilities of TO to TA phonon-assisted transitions is around 10 [17]. Moreover, the optical phonons emit and decay on a short time scale [5]. Based on these assumptions, we find that the short lifetime ( $\sim 400 \mathrm{fs})$ observed in our experiment is associated with the rapid decay of the TO phonon density and the longer lived component may result from the decay of the TA phonons generated by the excitation.

In order to obtain more quantitative information about the decay processes versus $t_{23}$ we choose different combinations of laser wavelengths. 

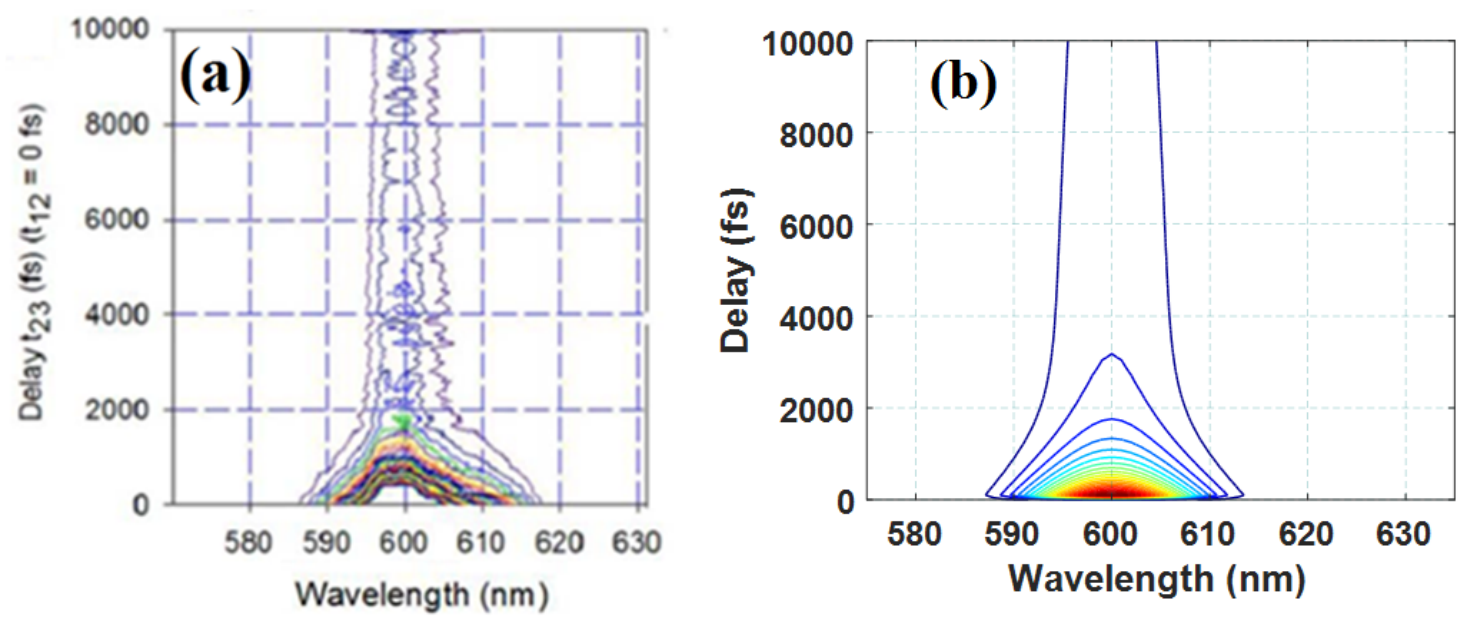

Fig. 2. Measured spectrum (a) and simulated spectrum (b) of three-pulse nonlinear signals $\left(\lambda_{1,2}=\lambda_{3}=600 \mathrm{~nm}\right)$ for Si QDs versus delay time $t_{23}$ up to $10 \mathrm{ps}$ when $t_{12}=0$


Fig. 3. Measured spectra (a) and simulated spectra (b) of three-pulse nonlinear signals $\left(\lambda_{1,2}=545 \mathrm{~nm}, \lambda_{3}=570 \mathrm{~nm}\right)$ for $\mathrm{Si}$ quantum dots versus short scan of population time $t_{23}$ when $t_{12}=0$.

Figure 3 shows the signal spectra versus delay $t_{23}$ for the wavelength of the two pump pulses at $545 \mathrm{~nm}$ and the wavelength of the probe pulse at $570 \mathrm{~nm}$. Interestingly, we observe a delay between transitions on the high and low energy sides in the experimental result shown in Fig. 3a. By performing a simulation (shown in Fig. 3b) and fitting to the experimental measurement, we are able to estimate this delay to be around $20 \mathrm{fs}$. 


\section{CONCLUSION}

We have demonstrated that two-color three-pulse spectrally resolved nonlinear spectroscopy can be used to study the carrier dynamics in semiconductor Si quantum dots. Our experimental results and theoretical simulations allow us to investigate different contributions of TO and TA phonon-assisted transitions to the carrier dynamics in this indirect band gap material.

\section{REFERENCES}

[1] H. Zewail, Femtochemistry: Ultrafast Dynamics of the Chemical Bond, World Scientific, Singapore, 1994.

[2] T. H. Tievater, X. Li, D. G. Steel, D. Gammon, D. S. Kazer, D. Park, C. Piermarocchi, and L. J. Sham, Phys. Rev. Lett. 87 (2001) 133603.

[3] L. V. Dao, X. Wen, M. T. T. Do, P. Hannaford, E. Cho, Y. Cho and Y. Huang, J. Appl. Phys. 97 (2005) 013501.

[4] N. H. Bonadeo, J. Erland, D. Gammon, D. Park, D. S. Katzer and D. G. Steel, Science 282 (1998) 1473.

[5] L. V. Dao, M. Lowe, P. Hannaford, H. Makino, T. Takai, and T. Yao, Appl. Phys. Lett. 81 (2002) 1806.

[6] D. M. Jonas, Annu. Rev. Phys. Chem. 54 (2003) 425.

[7] M. Woerner, W. Kuehn, P. Bowlan, K. Reimann and T. Elsaesser, New J. Phys. 15 (2013) 025039.

[8] G. Moody and S. T. Cundiff, Adv. Phys. X. 2 (2017) 641.

[9] M. Richter, R. Singh, M. Siemens and S. T. Cundiff, Sci. Adv. 4 (2018) eaar7697.

[10] L. Bruder, U. Bangert, M. Binz, D. Uhl, R. Vexiau, N. B.-Maafa, O. Dulieu and F. Stienkemeier, Nat. Commun. 9 (2018) 4823.

[11] L. Bruder, U. Bangert, M. Binz, D. Uhl and F. Stienkemeier, J. Phys. B: At. Mol. Opt. Phys. 52 (2019) 183501.

[12] R. Lopez-Delgado et al., Sci. Rep. 7 (2017) 14104.

[13] J. Haschke, O. Dupré, M. Boccard and C. Ballif, Sol. Energy Mater. Sol. Cells 187 (2018) 140.

[14] L. V. Dao, C. Lincoln, M. Lowe and P. Hannaford, J. Chem. Phys. 120 (2004) 8434.

[15] L. V. Dao, J. Davis, P. Hannaford, Y.Cho, M. A. Green and E. Cho, Appl. Phys. Lett. 90 (2007) 081105.

[16] C. W. Jiang and M. A. Green, J. Appl. Phys. 99 (2006) 114902.

[17] C. Delerue, G. Allan and M. Lannoo, Phys. Rev. B 64 (2001) 193402. 